\title{
Nanopolymers Delivery of the Bone Morphogenetic Protein-4 Plasmid to Mesenchymal Stem Cells Promotes Articular Cartilage Repair In Vitro and In Vivo
}

\author{
Junjun Shi, ${ }^{1}$ Xin Zhang, ${ }^{1}$ Yanbin Pi, ${ }^{1}$ Jingxian Zhu, ${ }^{1}$ Chunyan Zhou, ${ }^{2}$ and Yingfang Ao ${ }^{1}$ \\ ${ }^{1}$ Institute of Sports Medicine, Peking University Third Hospital, Beijing 100191, China \\ ${ }^{2}$ Department of Biochemistry and Molecular Biology, School of Basic Medical Sciences, Key Laboratory of Molecular \\ Cardiovascular Sciences, Ministry of Education of China, Peking University, Beijing 100191, China
}

Correspondence should be addressed to Chunyan Zhou, chunyanzhou@bjmu.edu.cn and Yingfang Ao, yingfang.ao@gmail.com

Received 21 March 2012; Accepted 1 April 2012

Academic Editor: Haifeng Chen

Copyright ( $) 2012$ Junjun Shi et al. This is an open access article distributed under the Creative Commons Attribution License, which permits unrestricted use, distribution, and reproduction in any medium, provided the original work is properly cited.

\begin{abstract}
The clinical application of viral vectors for gene therapy is limited for biosafety consideration. In this study, to promote articular cartilage repair, poly (lactic-co glycolic acid) (PLGA) nanopolymers were used as non-viral vectors to transfect rabbit mesenchymal stem cells (MSCs) with the pDC316-BMP4-EGFP plasmid. The cytotoxicity and transfection efficiency in vitro were acceptable measuring by CCK-8 and flow cytometry. After transfection, Chondrogenic markers (mRNA of Col2a1, Sox9, Bmp4, and Agg) of experimental cells (MSCs being transfected with BMP-4 plasmid by PLGA nanopolymers) were increased more than those of control cells (MSCs being transfected with naked BMP-4 plasmid alone). In vivo study, twelve rabbits (24 knees) with large full thickness articular cartilage defects were randomly divided into the experimental group (MSCs being transfected with BMP-4 plasmid by PLGA nanopolymers) and the control group (MSCs being transfected with naked BMP-4 plasmid). The experimental group showed better regeneration than the control group 6 and 12 weeks postoperatively. Hyaline-like cartilage formed at week 12 in the experimental group, indicating the local delivery of BMP-4 plasmid to MSCs by PLGA nanopolymers improved articular cartilage repair significantly. PLGA nanopolymers could be a promising and effective non-viral vector for gene therapy in cartilage repair.
\end{abstract}

\section{Introduction}

Full-thickness articular cartilage defects in the knee are still a major problem in orthopedics because of the limited repair capacity of cartilage. These defects may result in pain, swelling, and hopping, and they may even progress to osteoarthritis. The prevalence of cartilage defects is reported to $60 \%$ in the knee arthroscopy patients worldwide [1]. Recent studies of cartilage repair have focused on tissue engineering and gene therapy.

Bone morphogenetic protein 4 (BMP-4) has attracted much attention in cartilage repair in recent years because it accelerates chondrogenesis, the deposition of both cartilage matrix and type II collagen, chondrocyte differentiation, matrix maturation, and cartilage regeneration in vitro and in vivo [2-5].

For gene delivery, viral vectors such as adenovirus and retrovirus have been frequently used and extensively investigated. But their clinical applications are limited for safety considerations. Though high transfection efficiency, these viral vectors pose the risk of recombining to regenerate wild-type, replication-competent strains and have, at some point, been associated with or caused death $[6,7]$. Nonviral gene vectors such as lipoplexes, polyplexes, oligonucleotides and nanopolymers have attracted much attention recently [8]. With the rapid development of nanotechnology, there is increasing interest in nanopolymers for gene delivery because they have low immunogenicity, genotoxicity, oncogenicity and potential pathogenicity. These gene vectors are nontoxic and will not integrate into the host genome, thereby avoiding many of the risks of viral vectors. Many nanopolymers such as polyethyleneimine and poly (amido amine) dendrimers have been reported as gene delivery vectors [9-11].

However, few studies have reported on nonviral nanopolymers as gene vectors for cartilage repair. 
Poly(lactic-co-glycolic acid) (PLGA) is biodegradable and has been approved by the Food and Drug Administration (FDA) for clinical applications. NanoFect (QIAGEN, Germany) is a commercial reagent based on modified PLGA nanotechnologies [12]. They are chemically synthesized, lipid-free, free of animal-derived components and lack endotoxins. They are composed of ultrasmall nanopolymers. They can form transfection complexes in the nanometer size range when mixed with DNA. In this study, we used this kind of commercial PLGA nanopolymers to deliver the BMP-4 plasmid DNA into MSCs to evaluate their effect both in vitro and in vivo.

\section{Methods}

2.1. Animals. Male Japanese White Rabbits (age 3-4 months, weight $3-3.5 \mathrm{~kg}$ ) were obtained from the Animal Department of Peking University Health Science Center. All experiments were approved by the local Institutional Animal Care and Use Committee and complied with the Guide for the Care and Use of Laboratory Animals (1996).

2.2. Cell Isolation and Culture. The MSCs used in this study were isolated from the rabbit's femur bone marrow. Briefly, under sterile condition, femurs were excised. Bone marrow was flushed from the marrow cavity by PBS and was fractionated over a Ficoll-Paque (Dingguo, Beijing, China). Then the mononuclear cells were plated in $10 \mathrm{~mm}$ plates in Dulbecco's Modified Eagle Medium-(DMEM-) low glucose (GIBCO, Gaithersburg, USA) supplemented with $10 \%$ fetal bovine serum (HyClone, Logan, USA). The nonadherent cells were removed by changing the medium. The cultures were maintained at $37^{\circ} \mathrm{C}$ in a humidified incubator containing 5\% $\mathrm{CO}_{2}$ and the mediums were changed every 3 days.

2.3. Cell Surface Markers. CD44, CD90, CD14, and CD45 (antibodies from AbD Serotec, UK) on the surface of MSCs were analyzed by flow cytometry (FACS). Anti-mouse IgG1 was used as an isotype control.

After reached a confluence of $90 \%$, the ADSCs were detached from the flasks by incubation with $0.2 \%$ trypsin for 30 seconds. ADSCs of $1 \times 10^{6}$ cells were suspended by $100 \mu \mathrm{L}$ PBS with $10 \mu \mathrm{L}$ labeled mAb specific for CD44 (MCA806G), CD90 (MCA47R), CD45 (MCA808G), or $10 \mu \mathrm{L}$ conjugated $\mathrm{mAb}$ directed against CD14 (MCA2804C). After incubation, cells were washed three times with $2 \mathrm{~mL}$ PBS. Binding of the primary $\mathrm{mAb}$ was visualized using FITC-labeled secondary antibody (Zhongshan, Beijing, China) in a working dilution of $1: 100$. Mouse IgG1 (MCA1209C or MCA1209F) was used as an isotype control. All the antibodies were purchased from AbD Serotec (Oxford, UK). Finally, cells were washed with PBS and the membrane fluorescence was analyzed by flow cytometry analysis. The experiments were triplicated for verification.

2.4. Plasmid. Plasmid pDC316-BMP4-EGFP used in this study, containing the coding sequence of the enhanced Green
Fluorescent Protein (EGFP) and BMP-4, was constructed by Vector Gene Technology Company (Beijing, China). DNA sequence encoding BMP-4 was generated from plasmid pAM/CAG-BMP4 (gift from Dr Yan CHAN, Hospital for Sick Children, University of Toronto, Ontario, Canada) by PCR and subcloned into the NotI-HindIII sites of the pDC316-mCMV-EGFP vector. The construct was verified by DNA sequencing.

In studies of in vivo, experimental cells (MSCs being transfected with BMP-4 plasmid by PLGA nanopolymers) and control cells (MSCs being transfected with naked BMP-4 plasmid alone) were both tested and compared with.

2.5. Cell Viability. Cell viability was measured by Cell Counting Kit-8 (CCK-8, Dojindo, Kumamoto, Japan) at 72 hours after transfection. MSCs were seeded in 96-well plates at $5 \times 10^{3}$ cells $/ 100 \mu \mathrm{L}$ medium per well and incubated for 24 hours to allow attachment. The transfection procedures were performed according to the instruction manual. BMP4 plasmid DNA $(0.25 \mu \mathrm{g})$ was condensed with different amounts of PLGA nanopolymers (NanoFect) $(0.75 \mu \mathrm{L}, 1 \mu \mathrm{L}$ and $1.25 \mu \mathrm{L}$ ) giving rise to PLGA nanopolymers with PLGA nanopolymers/DNA (N/D; v/w) ratios of 3,4 , and 5, respectively. Then the nanopolymers/DNA complex was added to the experimental cells, while the BMP-4 naked plasmid DNA $(0.25 \mu \mathrm{g})$ alone was added to the control cells per well. After 24 hours, $10 \mu \mathrm{L}$ CCK-8 was added per well. The absorbance at $450 \mathrm{~nm}$ was measured using a microplate reader (BioRad, Tokyo, Japan) one hour later.

2.6. Transfection Efficiency. MSCs were seeded in 6-well plates at $3 \times 10^{5} / 2 \mathrm{~mL}$ medium per well and incubated for 24 hours to allow attachment. Then the BMP-4 plasmid $(7.5 \mu \mathrm{g})$, being condensed with PLGA nanopolymers at N/D ratios of 3,4 and 5 in $30 \mu \mathrm{L}$ medium, was added to the experimental cells per well. The BMP-4 plasmid $(7.5 \mu \mathrm{g})$ alone was added to the control cells per well. Transfection efficiency was evaluated by EGFP expressing using FACS 72 hours after transfection.

The best N/D ratio of 4 , being determined from the cytotoxicity and transfection experiments, was used in subsequent experiments.

2.7. Immunofluorescence Analysis. After 72 hours of transfection, cells were fixed, perforated, and blocked by $1 \%$ BSA. BMP-4 antibody (sc-12721, Santa Cruz, CA, USA) was incubated at 1:50 for 2 hours, followed by incubation with a 1:100 TRITC-labeled secondary antibody (Zhongshan, Beijing, China) for 30 minutes. Nuclei were stained by Hoechst 33258 (blue, 1:1000, Invitrogen, CA, USA) for 15 minutes. The cells were observed under a laser confocal microscope.

2.8. Real-Time RT-PCR. After 3, 7, and 10 days of transfection, total RNA of MSCs was extracted and reverse transcribed to cDNA. Real-time RT-PCR was performed using SYBR Green Real-time PCR Master Mix (TOYOBO, Osaka, Japan) according to the manufacturer's instructions 
by real-time RT-PCR instrument. The sequence of primers for GAPDH, type II collagen, and aggrecan had been previously described [13-15]: GAPDH: 5'-TCACCATCTTCCAGGAGCGA-3', 5'-CACAATGCCGAAGTGGTCGT$3^{\prime}$; type II collagen (Col2a1): 5'-AACACTGCCAACGTCCAGAT-3', 5'-CTGCAGCACGGTATAGGTGA-3'; Aggrecan (Agg): $5^{\prime}$-GCTACGGAGACAAGGATGAGTTC-3', 5' CGTAAAAGACCTCACCCTCCAT-3'; Sox $9: 5^{\prime}$-AGTACCCGCACCTGCACAAC- $3^{\prime}, 5^{\prime}$-CGCTTCTCGCTCTCGTTCAG-3'; Bmp4: 5'-ATGTGACACGGTGGGAAACTTTC-3', $5^{\prime}$-ACCTCAATGGCCAGCCCATA-3'. All gene expression was normalized to expression of the GAPDH. Data are presented in experimental cells as the fold change over the control cells. Three independent experiments were performed.

2.9. Scanning Electronic Microscopy (SEM) Observation of Scaffold. Cells after transfection 72 hours were seeded onto the PLLGA scaffold (Synthecon, Houston, USA) at a density of $1 \times 10^{6}$ cells $/ \mathrm{mL}$ and cultured in medium for another 3 days. Then the MSCs-PLLGA composition was fixed immediately in $4 \mathrm{~mL} 2.5 \%$ glutaraldehyde at $4^{\circ} \mathrm{C}$ for 4 hours, dehydrated in ethanol, critical-point dried from liquid $\mathrm{CO}_{2}$, coated with gold, and viewed with a scanning electron microscope (JEOL, JSM-5600LV, Tokyo, Japan).

2.10. Full-Thickness Articular Cartilage Defect Repair. After 12 rabbits were anesthetized intravenously with $2.5 \%$ pentobarbital sodium $(1 \mathrm{~mL} / \mathrm{kg})$, full-thickness cylindrical cartilage defects $(4.5 \mathrm{~mm}$ in diameter, $0.8 \mathrm{~mm}$ in depth) were created in the femoral trochlea of both knees of each rabbit using a corneal trephine under sterile condition. The two knees of each rabbit were randomly divided into experimental group or control group. The PLLGA scaffolds were made the same shape and size with the defect. The specific PLLGA scaffolds which were implanted for each group were prepared as follows: the experimental group, PLLGA scaffolds being seeded with experimental cells (MSCs being transfected with BMP-4 plasmid by PLGA nanopolymers 48 hours) and cultured for another one day; the control group, PLLGA scaffolds being seeded with control cells (MSCs being transfected with naked BMP-4 plasmid alone 48 hours) and being cultured for another one day. Incisions were closed in layers. The rabbits were kept in cages allowing full freedom of movement without immobilization. Every three rabbits in each group were sacrificed at 6 weeks and 12 weeks after surgery. One rabbit was sacrificed to observe the cells and the scaffold one week after surgery.

2.11. Macroscopic and Microscopic Observation. The distal femurs were removed and fixed in $4 \%$ paraformaldehyde ( $\mathrm{pH} 7.4$ ), decalcified in 10\% EDTA ( $\mathrm{pH} 7.2$ ), and embedded in paraffin. Serial paraffin sections of $6 \mu \mathrm{m}$ were cut along the maximum diameter of the repaired sites and stained with hematoxylin and eosin (HE) and with toluidine blue. Immunohistochemistry was performed with antibody for type II collagen (dilution 1:200, CP18, Calbiochem, CA, USA).
TABle 1: Pineda cartilage repair score.

\begin{tabular}{|c|c|}
\hline Characteristics & Score \\
\hline \multicolumn{2}{|l|}{ Filling of defect } \\
\hline $125 \%$ & -1 \\
\hline $100 \%$ & 0 \\
\hline $75 \%$ & 1 \\
\hline $50 \%$ & 2 \\
\hline $25 \%$ & 3 \\
\hline $0 \%$ & 4 \\
\hline \multicolumn{2}{|l|}{$\begin{array}{l}\text { Reconstruction of osteochondral } \\
\text { junction }\end{array}$} \\
\hline Yes & 0 \\
\hline Almost & 1 \\
\hline Not close & 2 \\
\hline \multicolumn{2}{|l|}{ Matrix staining } \\
\hline Normal & 0 \\
\hline Reduced staining & 1 \\
\hline Significantly reduced staining & 2 \\
\hline Faint staining & 3 \\
\hline No stain & 4 \\
\hline \multicolumn{2}{|l|}{ Cell morphology } \\
\hline Normal & 0 \\
\hline Most hyaline and fibrocartilage & 1 \\
\hline Mostly fibrocartilage & 2 \\
\hline Some fibrocartilage, but mostly & 3 \\
\hline Nonchondrocytic cells & 4 \\
\hline
\end{tabular}

A score of 0 indicates normal cartilage, with score of 12 indicating the most severe cartilage defects.

2.12. Histological Scores. The Pineda cartilage repair score was used to evaluate the results of the cartilage regeneration (Table 1). The score was ranged from 0 to 12 . A score of 0 indicates normal cartilage, with higher scores indicating more severe cartilage defects. The samples were measured by two observers who were blinded to the group identities.

2.13. Statistical Analysis. All in vitro experiments were repeated three times. The data of Pineda scores were presented as medians \pm interquartile range and analyzed with Mann-Whitney test. The data of others were presented as the means \pm standard deviation and analyzed with ANOVA followed by post hoc Tukey's test. SPSS 17.0 was used for statistical analysis. Differences were considered to be statistically significant at $P<0.05$.

\section{Results}

3.1. Surface Markers of MSCs. The MSCs surface markers were examined by FACS. MSCs were positive for the surface markers CD44 and CD90 (99.17\% and 93.37\%); negative for typical hematopoietic of $\mathrm{CD} 14$ and $\mathrm{CD} 45$, indicating that they were of mesenchymal origin (Figure 1(a)). 

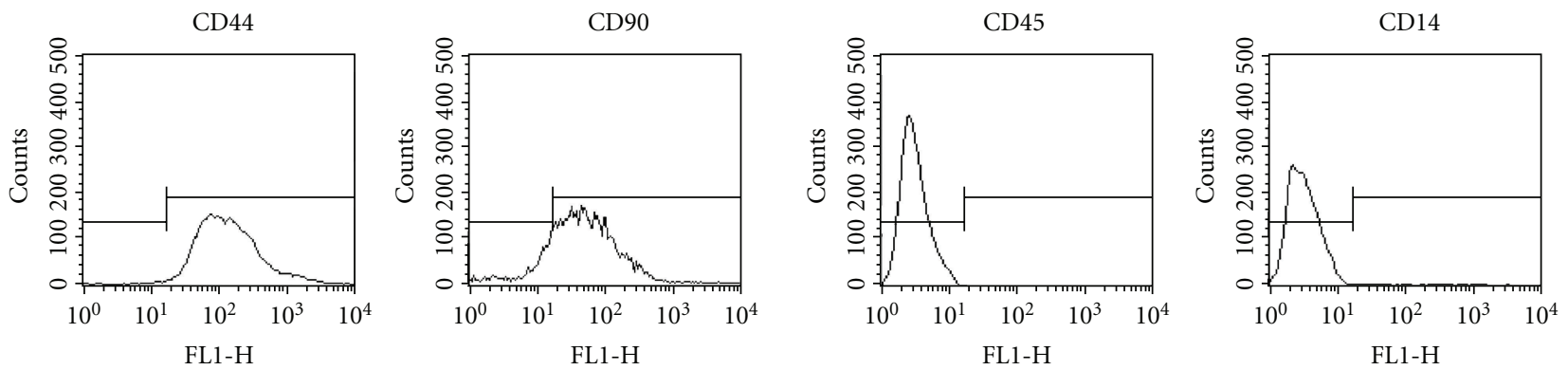

(a)

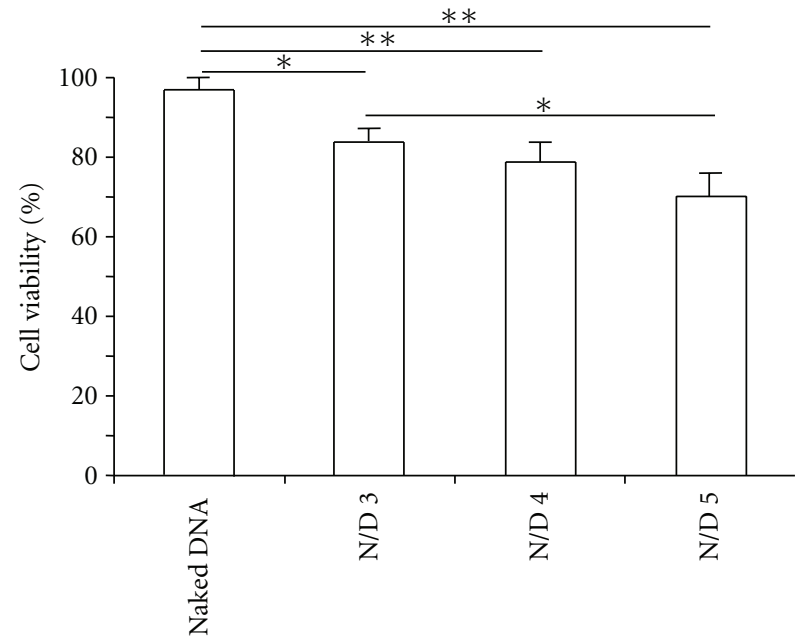

(b)

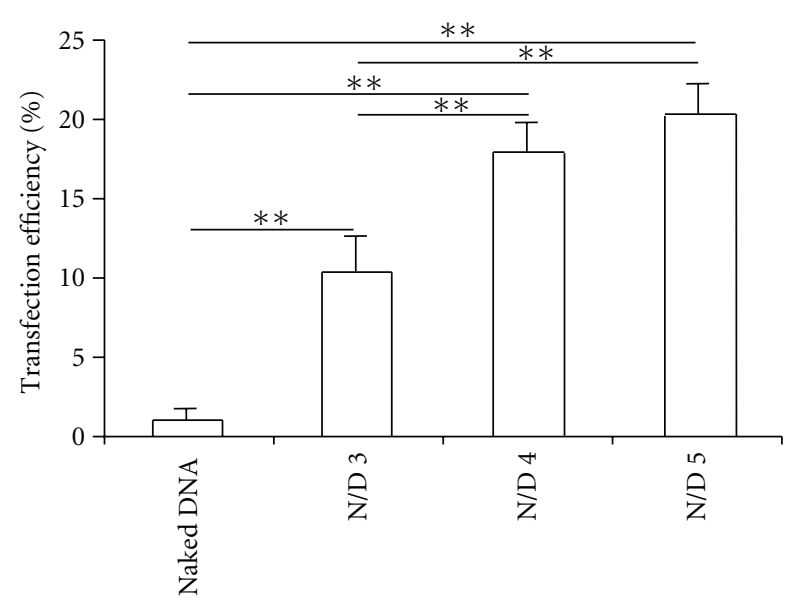

(c)

FIGURE 1: MSCs surface markers (a), cytotoxicity (b), and transfection efficiency (c) of PLGA nanopolymers. The mesenchymal-related antigens CD44 and CD90 were positive, but the hematopoietic-related antigens CD14 and CD45 were negative (a). The cell viability of the experimental cells (MSCs being transfected with BMP-4 plasmid by PLGA nanopolymers) significantly, decreased than that of the control cells (MSCs being transfected with naked BMP-4 plasmid alone) 72 hours after transfection. The cell viability was decreased with the N/D ratio increasing (b). The transfection efficiency of PLGA nanopolymers significantly increased than that of naked BMP-4 plasmid alone 72 hours after transfection, and was increased with the N/D ratio increasing (c). ${ }^{*} P<0.05,{ }^{* *} P<0.01$.

3.2. Cytotoxicity and Transfection Efficiency. The cytotoxicity of the PLGA nanopolymers to MSCs was tested by CCK8. Results showed an increasing trend of cytotoxicity with the increasing N/D ratio 72 hours after transfection. The BMP-4 plasmid was kept $0.25 \mu \mathrm{g}$ constant per well in the 96well plate. There were statistical differences between groups $(P<0.01)$. The cytotoxicity of the nanopolymers/DNA complex was significantly higher than that of the naked BMP-4 plasmid alone $(P<0.05)$. There was a statistically significant increase in cytotoxicity at N/D 5 than that at N/D $3(P<0.05)$, but the difference between N/D 3 and N/D 4 was not statistically significant, as well as between N/D 4 and N/D $5(P>0.05)$ (Figure 1(b)).

Transfection efficiency was analyzed for EGFP by FACS. Results showed an increasing trend of transfection efficiency with the increasing N/D ratio 72 hours after transfection. There were statistical differences between groups $(P<0.01)$. The transfection efficiency of the nanopolymers/DNA complex was significantly higher than that of naked BMP-4 plasmid alone $(P<0.01)$. The transfection efficiency of N/D 3 was statistically significant lower when than that of N/D 4 or N/D $5(P<0.01)$. There was a statistically significant increase in transfection efficiency at N/D 5 than at N/D 3 $(P<0.05)$, but the difference of the transfection efficiency between N/D 3 and N/D 4 was not statistically significant, as well as between N/D 4 and N/D $5(P>0.05)$ (Figure 1(c)).

3.3. BMP-4 Expression. Immunofluorescence was used to detect the BMP-4 expression after transfection. After 72 hours, both strong green fluorescence (EGFP) and red light (TRITC-labeled BMP-4 antibodies) were observed in the experimental cells, indicating EGFP and BMP-4 expression by a fluorescence microscope. However, EGFP and BMP-4 were not detected in the control cells (Figure 2(a)).

3.4. mRNA Expression. To detect the expression of chondrogenic markers, mRNA were measured by real-time RTPCR. The mRNA levels of Bmp4, Sox9, Agg, and Col2a1 in experimental cells were significantly upregulated than that in the experimental cells at each time point $(P<0.05)$. The expression of Bmp4 mRNA remained constant at day 3 and day $7(P>0.05)$, then down-regulated at day 10 $(P<0.05)$. This indicated that the BMP-4 plasmid was 

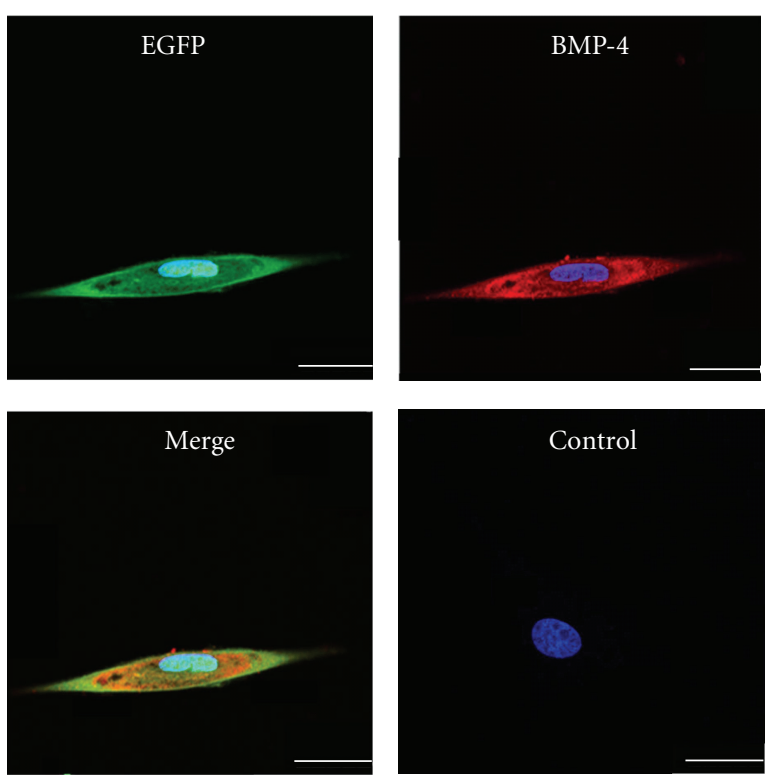

(a)

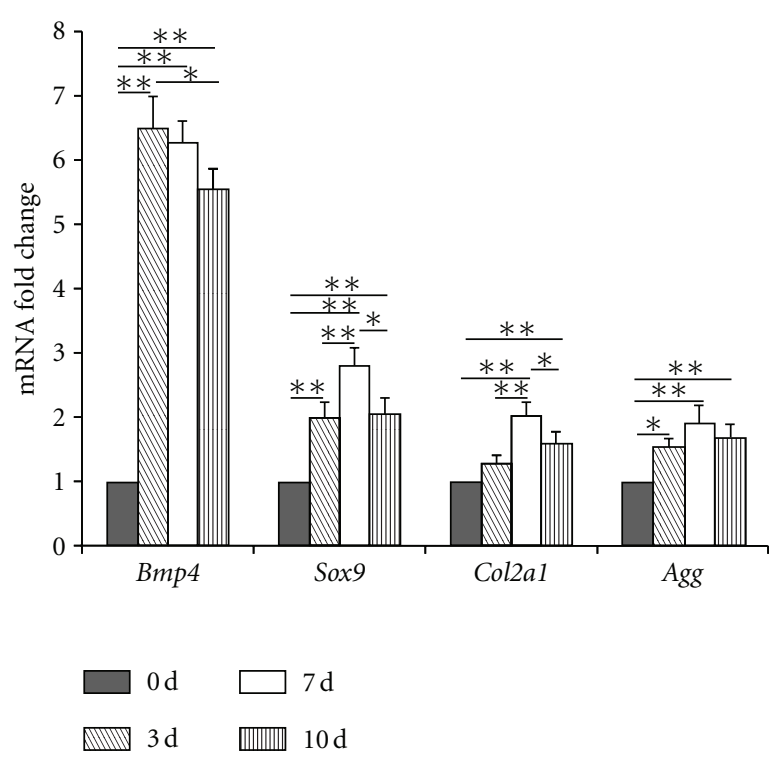

(b)

FIGURE 2: Immunofluorescence staining for BMP-4 (a) and the mRNA levels (b) of chondrogenic markers. EGFP (green) and BMP-4 (red) were expressed in the experimental cells (MSCs transfected with BMP-4 plasmid by PLGA nanopolymers). The control cells (MSCs being transfected with naked BMP-4 plasmid alone) showed no BMP-4 expression (a). The mRNA expressions of Bmp4, Sox9, Col2a1, and Agg in the experimental cells were upregulated in comparison with those of the control cells (b). ${ }^{*} P<0.05,{ }^{* *} P<0.01$.

transfected successfully and the expression could last for at least 10 days. The peak levels of Sox9, Agg, and Col2al mRNA were reached at day 7 after transfection and slowly decreased at day 10. This indicated the Bmp4 overexpression resulted in the increasing of Sox9, Agg, and Col2a1 mRNA (Figure 2(b)).

\subsection{SEM Observation of Scaffold-Cell Constructs. MSCs after} transfection three days were seeded into the PLLGA scaffold. The constructs were observed by SEM. MSCs were adhering to the scaffold and grew normally on the fibers of the scaffold (Figure 3).

3.6. Macroscopic Observations. No scaffold was dislodged from the defect site and no knee was infected after surgery. At week 6 , the defects in the experimental group were filled with some white, semitransparent tissue newly formed cartilage. The thickness of the newly cartilage was a little thinner than that of the surrounding normal cartilage, but there were still concavity toward the center. The margins were clear and the surface was not as smooth as the normal cartilage (Figure 4(a)). In the control group, semitransparent tissue of the new cartilage filled the defect. The thickness was thinner than the adjacent normal cartilage. The surfaces were rough and the margin were sharply defined (Figure 4(b)).

At week 12, in the experimental group, the regenerative tissue became thicker and smoother. The margins of the defects were unclear. The boundaries between the normal cartilage and the regenerative tissue were hard to recognize (Figure 4(c)). In the control group, the regenerative tissue become thicker and smoother than those at week 6 , but the centers were still a little thinner and uneven (Figure 4(d)).

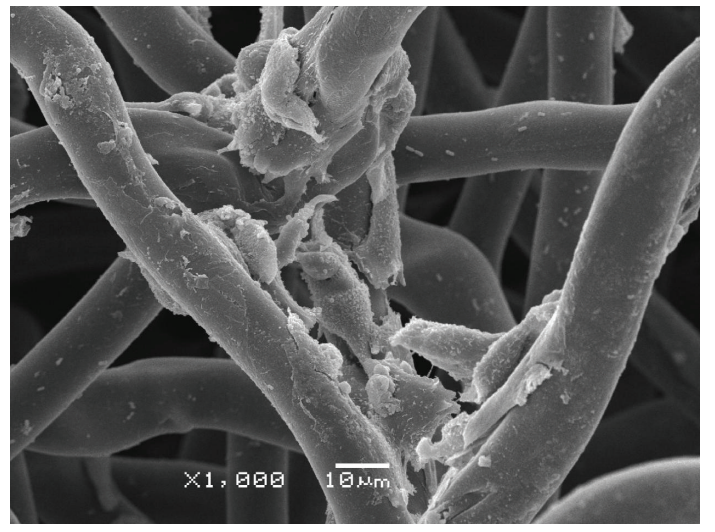

Figure 3: MSCs on PLLGA scaffolds. The MSCs adhered on the fibers of PLLGA scaffolds.

MSCs which were transfected with BMP-4 by PLGA nanopolymers stimulated articular cartilage regeneration more rapidly and effectively than the control ones.

3.7. Histological and Immunohistochemical Staining. At week 1 , sections were stained with HE. The PLLGA scaffolds were not absorbed. The fibers of PLLGA scaffold could be identified. The cells existed in the pores between the fibers of the scaffold (Figure 5).

Histological and immunohistochemical staining were performed and the results further demonstrated that MSCs which were transfected with BMP-4 by PLGA nanopolymers promotes cartilage regeneration (Figure 4).

At week 6, in the experimental group, the surfaces were smooth with thinner regenerated cartilage layers than the 
6 weeks

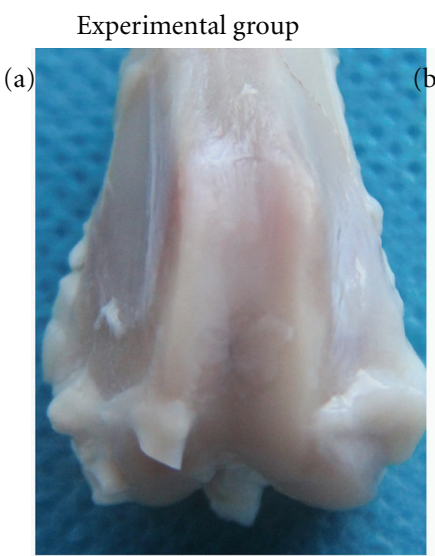

(e)

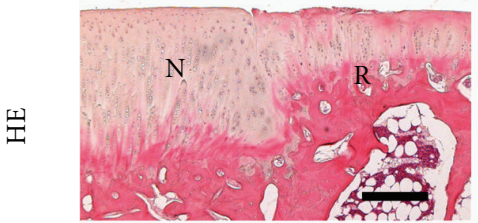

(i)

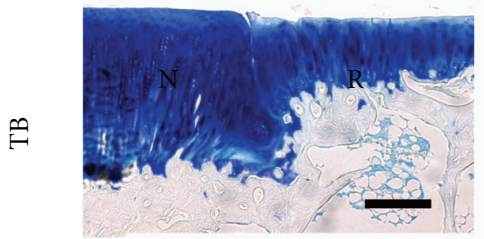

(j)

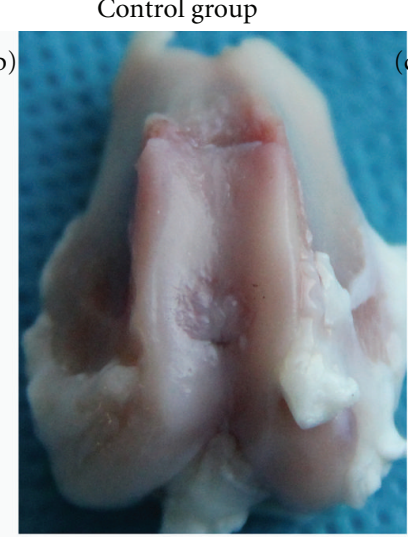

(f)

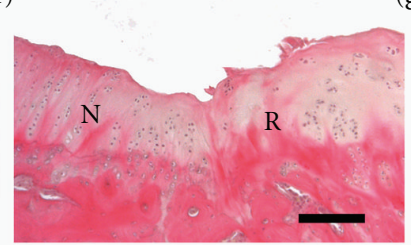

(g)
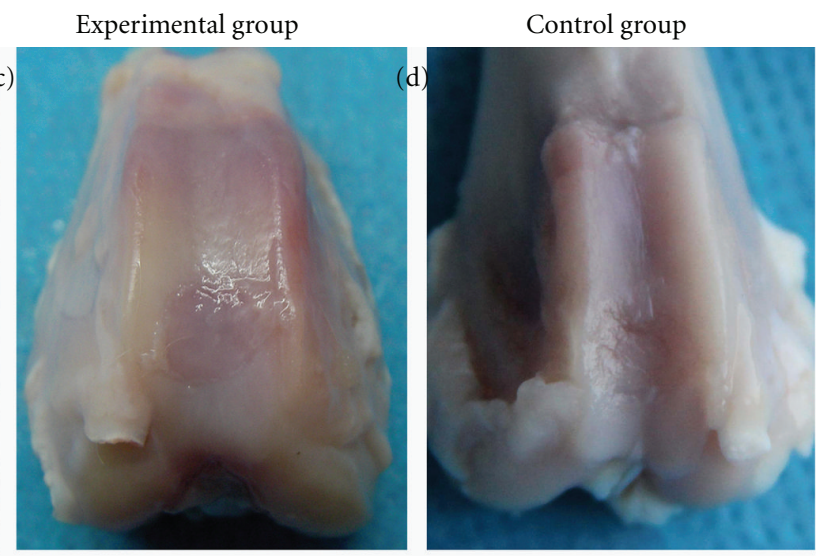

(h)
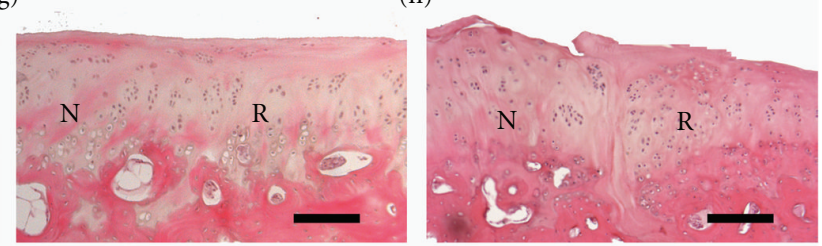

(k)

(1)
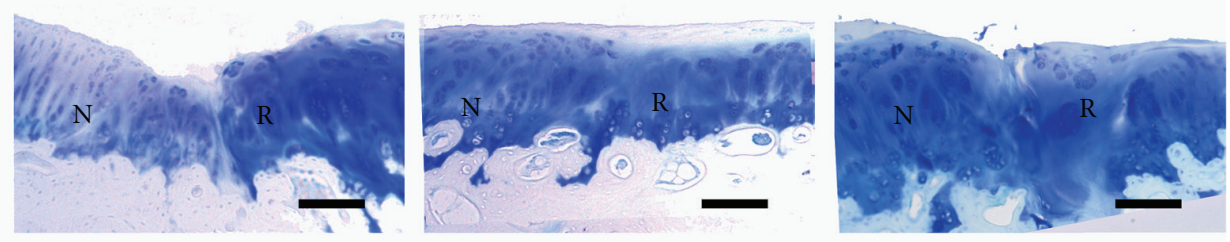

(m)

(n)

(o)

(p)
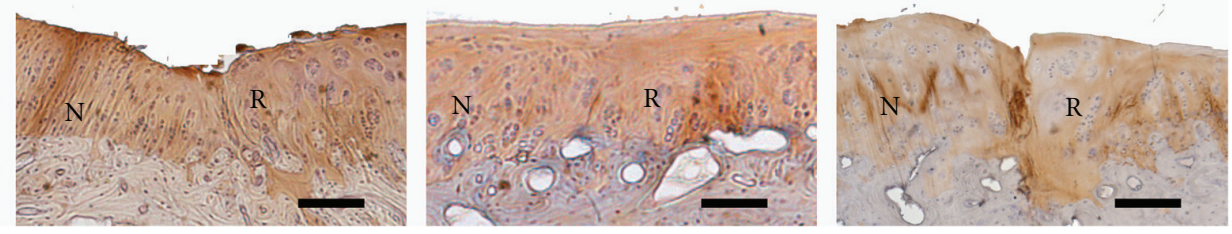

Figure 4: Macroscopic (a-d) and microscopic (e-p) observations of regenerated cartilage at week 6 and week 12. At week 6, in the experimental group, smooth semitransparent tissue newly tissues filled the defect, but there were still concavity toward the center. The new cartilages were stained positive with toluidine blue and collagen type II (a, e, i, m). In the control group, the thickness of the newly rough cartilage was thinner than normal cartilage. The junctions were obvious. Cells aligned areatus. Toluidine blue and collagen type II staining were positive $(b, f, j, n)$. At week 12, the thickness of the regenerative tissue in the experimental group was similar to the adjacent normal cartilage. The surfaces were regular and mostly integrated with the hosts. Aggrecan and collagen type II deposited (c, g, k, o). In the control group, the thickness of the newly cartilage layer was thinner than that of the host. The surfaces were uneven and the margins could be distinguished easily. Cells aligned irregularly. The excellular matrices were also formed because of the positive staining of toluidine blue and collagen type II (d, h, l, p). The bar was $200 \mu \mathrm{m}$. N refers to normal cartilage, R refers to regenerative cartilage.

host cartilage. Cells aligned columnar, but few cartilage lacunae could be seen. The newly cartilages were stained positive with toluidine blue and collagen type II, indicating the extracellular matrix became formed (Figures 4(e), 4(i), and $4(\mathrm{~m})$ ). In the control group, the surfaces were uneven. The junctions were crimp and there was lack of thickness. Cells aligned areatus. Toluidine blue and collagen type II staining were positive in the new tissues (Figures $4(\mathrm{f}), 4(\mathrm{j})$, and $4(\mathrm{n}))$.

At week 12, in the experimental group, the thickness was almost the same as that of the adjacent cartilage. The surfaces usually regular and mostly integrated with the host, but margins still could be identified. Toluidine blue and collagen type II staining were similar to the surrounding cartilages (Figures $4(\mathrm{~g}), 4(\mathrm{k})$, and $4(\mathrm{o})$ ). In the control group, the thickness of the new cartilage layers were a little thinner than that of the hosts. The surfaces were uneven and the margins could be distinguished easily. The cells aligned irregularly. The excellular matrices were also formed because of the positive staining of toluidine blue and collagen type II (Figures 4(h), 4(l), and 4(p)).

3.8. Pineda Scores. Pineda cartilage repair score was used to evaluate the effect of cartilage repair. The scores of the 


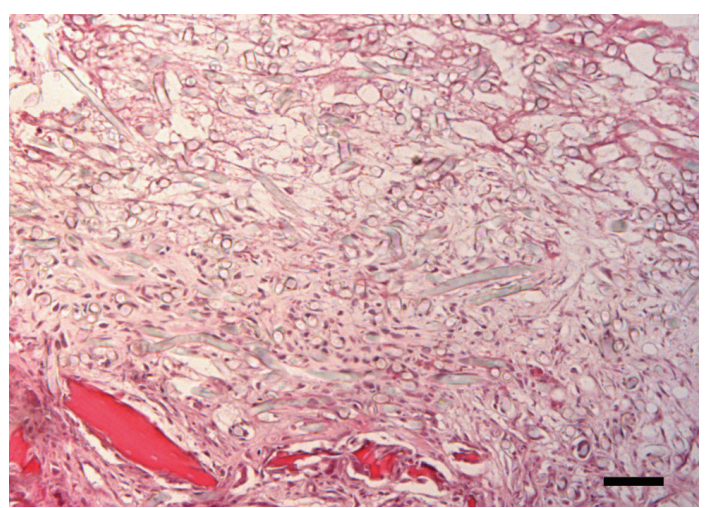

Figure 5: Microscopic observations (HE) of the PLLGA scaffold being implanted at week 1. The fibers of PLLGA scaffold could be identified. The cells existed in the pores between the fibers of the scaffold. The bar was $200 \mu \mathrm{m}$.

experimental group were significant lower than that of the control group at both week $6(5.83 \pm 1.85$ versus $7.91 \pm 1.48)$ and week $12(1.85 \pm 1.56$ versus $5.75 \pm 1.14)(P<0.05)$. We could also find the scores of both groups at week 12 were lower than those at week $6(P<0.05)$ (Figure 6).

\section{Discussion}

The aim of this study was to optimize nanopolymers for transfection of rabbit MSCs. We isolated rabbit MSCs, detected their cell surface markers. Then we evaluated their cytotoxicity, transfection efficiency, and mRNA expression after transfection by PLGA nanopolymers in vitro. The cartilage repair effect of MSCs transfected with BMP-4 by PLGA nanopolymers was also evaluated in vivo. The results showed PLGA nanopolymers were safe and effective to deliver BMP-4 plasmid into MSCs and then the delivered plasmid could play its role on cartilage regeneration.

The transfection efficiency of viral vectors is significant, but the potential pathogenicity and oncogenicity could not be ignored. Nonviral methods are commonly less effective for gene transfer to MSCs [16]. Naked plasmid DNA has poor transfection efficiency because of their large size and hydrophilic nature due to negatively charged phosphate groups [17]. In addition, they are very susceptible to nuclease-mediated degradation [17]. Thus, nonviral vectors are favored. As nanotechnology advances rapidly, many types of nanoparticles have been used to deliver drugs, proteins and DNA into cells. NanoFect is a commercial nanovector. Report shows NanoFect transfection reagent is based on modified PLGA nanopolymers [12]. In our study, we select NanoFect as the gene vector because PLGA has been approved by the FDA for clinical use and commercial reagents are stable and feasible for experiments repeating. Studies have shown that betamethasone sodium phosphate contained in PLGA nanoparticles can be delivered to chondrocytes. The drug release from the nanopolymers is controlled by the molecular weight and the PLA/PGA ratio of the polymers, which allows the effective period of the DNA or protein to be adjusted [18]. Because its

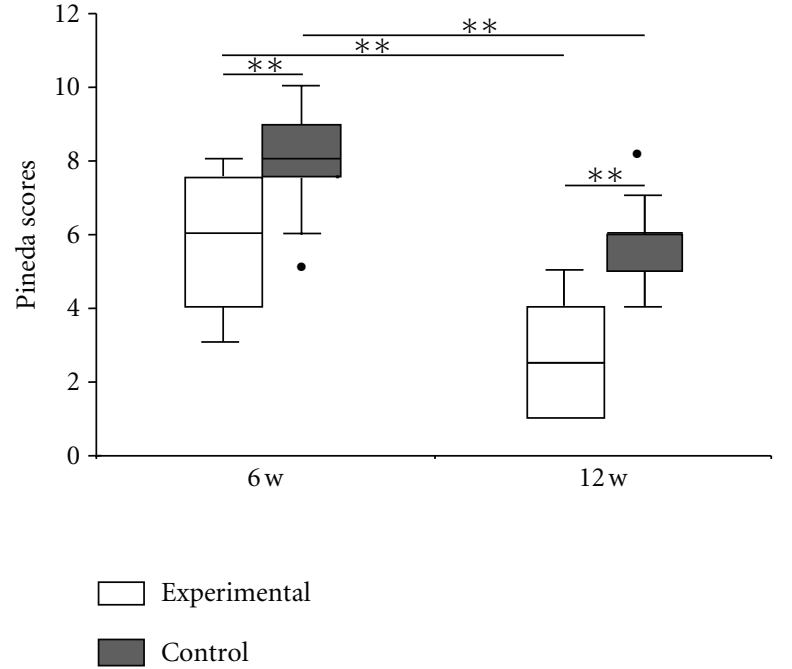

Figure 6: Pineda cartilage repair scores of the regenerative cartilage. Pineda cartilage repair score was used to evaluate the effect of cartilage repair. The scores of the experimental group were significant lower than that of the control group at either week 6 or week 12. We could also found the scores of both groups at week 12 were lower than that at week $6 .{ }^{*} P<0.05,{ }^{* *} P<0.01$.

properties are known, PLGA can be modified to have various characteristics for different uses and to target different cells and tissues. There were few studies about PLGA nanopolymers in gene transfer to MSCs. Kim et al. use PEImodified PLGA nanopolymers delivering SOX9 gene into human MSCs, showing satisfactory transfection efficiency at $99.38 \% 30 \mathrm{~min}$ to 4 hours after transfection [19]. Another relative study reported transfection efficiency of SOX9 gene into hMSCs with PEI-modified PLGA nanopolymers was $83 \%$ at two days after transfection [20]. The modification for NanoFect is not provided in the product instruction as a commercial secret. In our study, though the transfection efficiency we detected was not as high as these reports above $80 \%$, it was similar to that of Lipofectamine 2000 with the similar experimental conditions which were reported about $5 \% \sim 19.60 \%[21,22]$. Since MSCs were cells of difficult transfection, this transfection efficiency about 20\% was also acceptable. The difference between our study and the previous studies indicated surface modified was important for nanopolymers. Different modifications achieve notable results. Some more advance surface modification should be researched. Study also showed cytotoxicity lower than $10 \%$ after 72 hours [19]. Cytotoxicity of Lipofectamine 2000 was less than $20 \%[11,21]$. Results in this study showed the cytotoxicity was below $20 \%$ which was not so significant different from the reports.

Some study reports PLGA nanopolymers had little immunogenicity [23]. In our study, few inflammatory cells were observed in the experimental group in histological observations, demonstrating a low immunogenicity of the nanopolymers in vivo.

This study demonstrates that PLGA nanopolymers are able to deliver BMP-4 plasmid to MSCs effectively. The BMP4 plays an important role in chondrogenesis, extracellular 
matrix formation, and cartilage repair both in vitro and in vivo. The use of PLGA nanopolymers for gene delivery in cartilage repair has not been reported previously. BMP-4 has been the focus of much attention in recent years for its chondrogenic properties. Reports have shown that BMP-4 promotes chondrocyte differentiation, the extracellular matrix composition of cartilage, collage type II deposition, and cartilage regeneration [2-5]. Both BMP-4 protein and the BMP-4 plasmid transfected by viral vectors have been reported to stimulate chondrogenesis and repair cartilage defects $[24,25]$. In the present study, BMP-4 expression in mRNA and protein level in MSCs was observed at both 72 hours and 10 days after transfection. Results indicated that the plasmid being delivered by nanopolymers could last for at least 10 days. The chondrogenesis of BMP-4 was proven both in vitro and in vivo. After transfection, the expression of Bmp4 mRNA was upregulated and the secretion of BMP-4 protein was increased. Under the influence of BMP-4, the expression of the cartilage-specific markers Col2a1, Agg and Sox9 was upregulated; the secretion of collagen type II was increased. In vivo, the chondrogenesis effect of BMP-4 was also played. MSCs were induced to differentiate into chondrocytes by BMP-4. Then they were induced to secrete collagen type II and glycosaminoglycan. In the cartilage repair models, the regenerated cartilage in the experimental group showed better regeneration than the cartilage in the control group with respect to thickness, surface smoothness, and integration with the surrounding normal cartilage at the same time point. In our in vivo study, hyaline cartilage was regenerated at week 12 in the experimental group.

In cartilage regeneration researches, MSCs are one of the most frequently used stem cells. MSCs are cells can be self-renewed and induced to terminally differentiate into osteoblasts, chondrocytes, and adipocytes either in vitro or in vivo. Their easy isolation and culture, as well as their high in vitro expansive potential, make these cells an attractive therapeutic tool for tissue engineering and promising candidates for gene. Their self-renewal and multiplex differentiation potential have been reported previously. Moreover, compared with direct transfer of gene vectors, engineering MSCs are associated with less immunologic interference [16]. What is more, MSCs also have the capacity of enhancing their therapeutic potential. One of the hypothetical mechanisms is the paracrine effect. In fact MSCs can recognize the location of injury, reach the site, and excrete several soluble factors to accelerate the healing process [26]. MSCs seeding in vivo can compensate for the insufficient cells in cartilage. Correspondingly, the cartilage defects are repaired much better and faster in the groups treated with MSCs. Crosstalk between extracellular components in the microenvironment and MSCs within the cartilage further contributes to the differentiation of stem cells into chondrocytes [27]. The surface marker expression of MSCs we detected is generally consistent with that reported in the literature.

We used PLLGA scaffolds as a temporary mechanical support for cartilage regeneration. PLLGA is approved by the FDA for clinical applications. PLGA scaffolds are used in popular in tissue engineering [28-30]. The scaffold we used is of three-dimensional structure with the appropriate size pores which could provide space for cell living. Moreover, the PLLGA scaffolds had degraded in 6 weeks without disturbing the remodeling of the regenerated tissue.

\section{Conclusions}

Nanopolymers are promising nonviral vector for gene delivery displaying acceptable cytotoxicity and transfection efficiency. We used it to deliver BMP-4 plasmid DNA to MSCs and BMP-4 exerted its chondrogenic effects on the MSCs. After transfection, the expression of Sox9, Agg, and Col2a1 was upregulated and the collagen type II protein secretion was increased. MSCs transfected with BMP-4 plasmid using PLGA nanopolymers which were able to promote articular cartilage repair in vivo and to achieve hyaline-like cartilage regeneration in a short period. These findings suggest that BMP-4 plasmid transfection is a promising method for articular cartilage repair and that PLGA nanopolymers can serve as gene vectors for articular cartilage repair in vivo. This study provides an experimental basis for further clinical studies and applications.

\section{Acknowledgments}

This work was supported by the National Science and Technology Ministry of China (2007BAI04B09), the Program for Changjiang Scholars and Innovative Research Team in University (BMU2009129-112), and the Specialized Research Fund for the Doctoral Program of Higher Education (20100001110086).

\section{References}

[1] W. W. Curl, J. Krome, E. S. Gordon, J. Rushing, B. P. Smith, and G. G. Poehling, "Cartilage injuries: a review of 31,516 knee arthroscopies," Arthroscopy, vol. 13, no. 4, pp. 456-460, 1997.

[2] R. Kuroda, A. Usas, S. Kubo et al., "Cartilage repair using bone morphogenetic protein 4 and muscle-derived stem cells," Arthritis and Rheumatism, vol. 54, no. 2, pp. 433-442, 2006.

[3] N. Nakayama, D. Duryea, R. Manoukian, G. Chow, and C. Y. E. Han, "Microscopic cartilage formation with embryonic stem-cell-derived mesodermal progenitor cells," Journal of Cell Science, vol. 116, no. 10, pp. 2015-2028, 2003.

[4] J. Kramer, C. Hegert, K. Guan, A. M. Wobus, P. K. Müller, and J. Rohwedel, "Embryonic stem cell-derived chondrogenic differentiation in vitro: activation by BMP-2 and BMP-4," Mechanisms of Development, vol. 92, no. 2, pp. 193-205, 2000.

[5] X. Zhang, Z. Zheng, P. Liu et al., "The synergistic effects of microfracture, perforated decalcified cortical bone matrix and adenovirus-bone morphogenetic protein-4 in cartilage defect repair," Biomaterials, vol. 29, no. 35, pp. 4616-4629, 2008.

[6] S. Hacein-Bey-Abina, "LMO2-associated clonal T cell proliferation in two patients after gene therapy for SCID-X1," Science, vol. 302, no. 5644, pp. 415-419, 2003.

[7] T. Hollon, "Researchers and regulators reflect on first gene therapy death," Nature Medicine, vol. 6, no. 1, article 6, 2000.

[8] S. McLenachan, J. P. Sarsero, and P. A. Ioannou, "Flowcytometric analysis of mouse embryonic stem cell lipofection using small and large DNA constructs," Genomics, vol. 89, no. 6, pp. 708-720, 2007. 
[9] K. Kunath, A. Von Harpe, D. Fischer, and T. Kissel, "GalactosePEI-DNA complexes for targeted gene delivery: degree of substitution affects complex size and transfection efficiency," Journal of Controlled Release, vol. 88, no. 1, pp. 159-172, 2003.

[10] M. Huang, C. W. Fong, E. Khor, and L. Y. Lim, "Transfection efficiency of chitosan vectors: effect of polymer molecular weight and degree of deacetylation," Journal of Controlled Release, vol. 106, no. 3, pp. 391-406, 2005.

[11] J. L. Santos, E. Oramas, A. P. Pêgo, P. L. Granja, and H. Tomás, "Osteogenic differentiation of mesenchymal stem cells using PAMAM dendrimers as gene delivery vectors," Journal of Controlled Release, vol. 134, no. 2, pp. 141-148, 2009.

[12] Telomolecular Corporation, "Pre-Effective Amendment to Registration of Securities by a Small-Business Issuer. Form SB-2. In SEC," 2007, http://www.secinfo.com/d199pj.u6 .htm\#1stPage.

[13] H. Park, J. S. Temenoff, Y. Tabata, A. I. Caplan, and A. G. Mikos, "Injectable biodegradable hydrogel composites for rabbit marrow mesenchymal stem cell and growth factor delivery for cartilage tissue engineering," Biomaterials, vol. 28, no. 21, pp. 3217-3227, 2007.

[14] P. J. Emans, F. Spaapen, D. A. M. Surtel et al., "A novel in vivo model to study endochondral bone formation; HIF- $1 \alpha$ activation and BMP expression," Bone, vol. 40, no. 2, pp. 409418, 2007.

[15] C. J. Bowman, K. J. Turner, M. Sar, N. J. Barlow, K. W. Gaido, and P. M. D. Foster, "Altered gene expression during rat Wolffian duct development following Di(n-Butyl) phthalate exposure," Toxicological Sciences, vol. 86, no. 1, pp. 161-174, 2005.

[16] A. Van Damme, T. Vanden Driessche, D. Collen, and M. K. Chuah, "Bone marrow stromal cells as targets for gene therapy," Current Gene Therapy, vol. 2, no. 2, pp. 195-209, 2002.

[17] M. S. Al-Dosari and X. Gao, "Nonviral gene delivery: principle, limitations, and recent Progress," AAPS Journal, vol. 11, no. 4, pp. 671-681, 2009.

[18] E. Horisawa, T. Hirota, S. Kawazoe et al., "Prolonged anti-inflammatory action of DL-lactide/glycolide copolymer nanospheres containing betamethasone sodium phosphate for an intra-articular delivery system in antigen-induced arthritic rabbit," Pharmaceutical Research, vol. 19, no. 4, pp. 403-410, 2002.

[19] J. H. Kim, J. S. Park, H. N. Yang et al., "The use of biodegradable PLGA nanoparticles to mediate SOX9 gene delivery in human mesenchymal stem cells (hMSCs) and induce chondrogenesis," Biomaterials, vol. 32, no. 1, pp. 268278, 2011.

[20] J. S. Park, H. N. Yang, D. G. Woo et al., "Chondrogenesis of human mesenchymal stem cells mediated by the combination of SOX trio SOX5, 6, and 9 genes complexed with PEImodified PLGA nanoparticles," Biomaterials, vol. 32, no. 14, pp. 3679-3688, 2011.

[21] Y. Gheisari, M. Soleimani, K. Azadmanesh, and S. Zeinali, "Multipotent mesenchymal stromal cells: optimization and comparison of five cationic polymer-based gene delivery methods," Cytotherapy, vol. 10, no. 8, pp. 815-823, 2008.

[22] F. Yang, J. J. Green, T. Dinio et al., "Gene delivery to human adult and embryonic cell-derived stem cells using biodegradable nanoparticulate polymeric vectors," Gene Therapy, vol. 16, no. 4, pp. 533-546, 2009.

[23] B. Semete, L. I. J. Booysen, L. Kalombo et al., "In vivo uptake and acute immune response to orally administered chitosan and PEG coated PLGA nanoparticles," Toxicology and Applied Pharmacology, vol. 249, no. 2, pp. 158-165, 2010.

[24] R. Kuroda, A. Usas, S. Kubo et al., "Cartilage repair using bone morphogenetic protein 4 and muscle-derived stem cells," Arthritis and Rheumatism, vol. 54, no. 2, pp. 433-442, 2006.

[25] Y. Jiang, L. K. Chen, D. C. Zhu et al., "The inductive effect of bone morphogenetic protein-4 on chondral-lineage differentiation and in situ cartilage repair," Tissue Engineering, vol. 16, no. 5, pp. 1621-1632, 2010.

[26] F. Tögel, Z. Hu, K. Weiss, J. Isaac, C. Lange, and C. Westenfelder, "Administered mesenchymal stem cells protect against ischemic acute renal failure through differentiationindependent mechanisms," American Journal of Physiology, vol. 289, no. 1, pp. F31-F42, 2005.

[27] F. Djouad, B. Delorme, M. Maurice et al., "Microenvironmental changes during differentiation of mesenchymal stem cells towards chondrocytes," Arthritis Research and Therapy, vol. 9, article R33, 2007.

[28] W. S. Koegler and L. G. Griffith, "Osteoblast response to PLGA tissue engineering scaffolds with PEO modified surface chemistries and demonstration of patterned cell response," Biomaterials, vol. 25, no. 14, pp. 2819-2830, 2004.

[29] E. Lieb, S. Milz, T. Vogel, M. Hacker, M. Dauner, and M. B. Schulz, "Effects of transforming growth factor $\beta 1$ on bonelike tissue formation in three-dimensional cell culture: I. Culture conditions and tissue formation," Tissue Engineering, vol. 10, no. 9-10, pp. 1399-1413, 2004.

[30] E. Lieb, T. Vogel, S. Milz, M. Dauner, and M. B. Schulz, "Effects of transforming growth factor $\beta 1$ on bonelike tissue formation in three-dimensional cell culture. II: osteoblastic differentiation," Tissue Engineering, vol. 10, no. 9-10, pp. 14141425, 2004. 

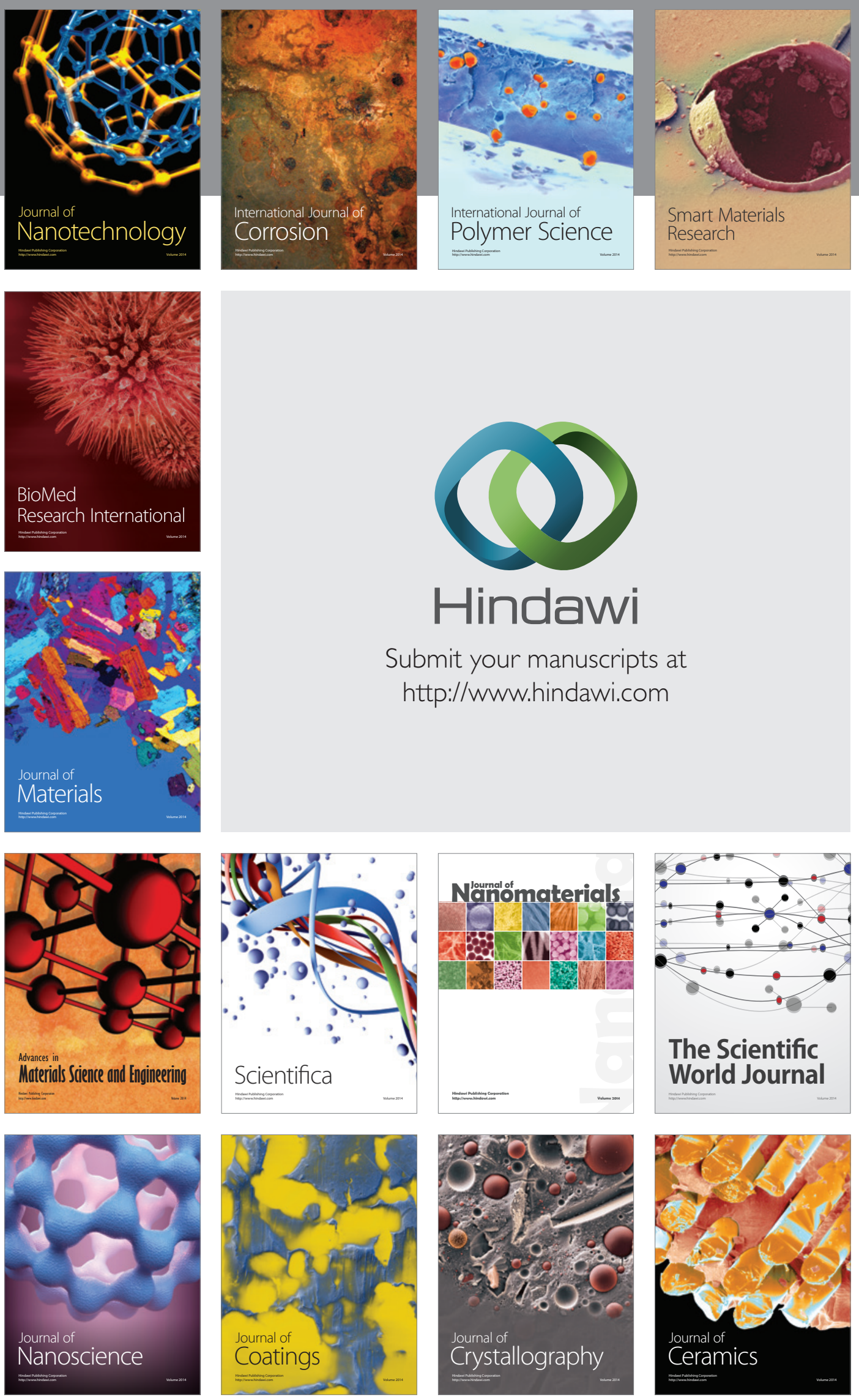

The Scientific World Journal

Submit your manuscripts at

http://www.hindawi.com

\section{World Journal}

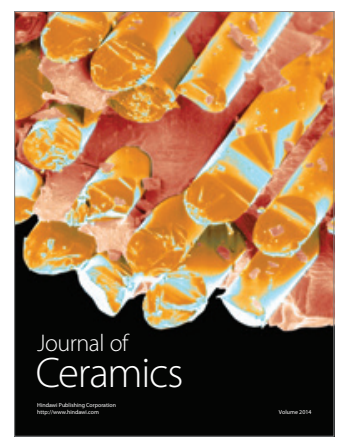

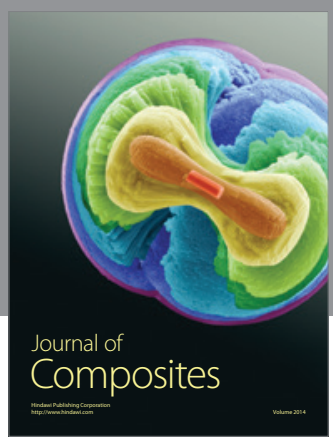
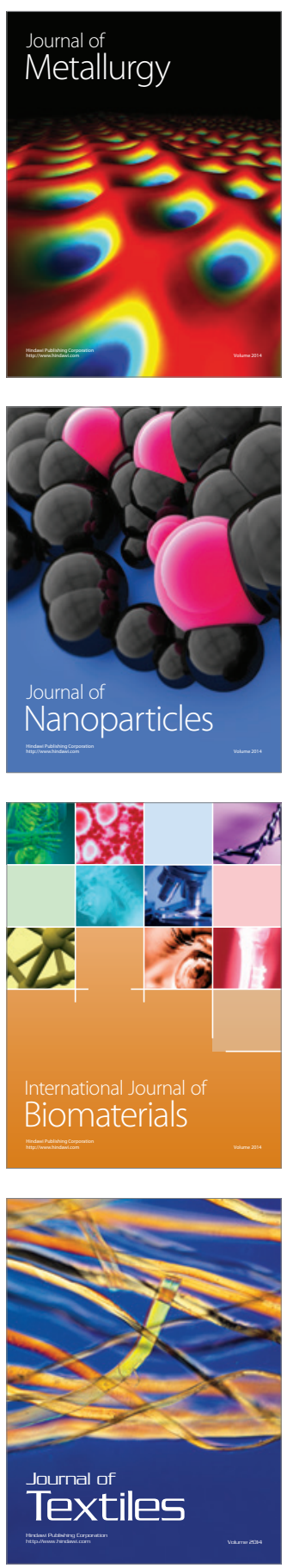